CORRECTION

https://doi.org/10.1038/s41586-018-0232-x

\title{
Author Correction: A molecular atlas of cell types and zonation in the brain vasculature
}

Michael Vanlandewijck, Liqun He, Maarja Andaloussi Mäe, Johanna Andrae, Koji Ando, Francesca Del Gaudio, Khayrun Nahar, Thibaud Lebouvier, Bàrbara Laviña, Leonor Gouveia, Ying Sun, Elisabeth Raschperger, Markus Räsänen, Yvette Zarb, Naoki Mochizuki, Annika Keller, Urban Lendahl \& Christer Betsholtz

Correction to: Nature https://doi.org/10.1038/nature25739, published online 14 February 2018.

In Fig. 1b of this Article, 'Csflr' was misspelt 'Csfrl'. In addition, in Extended Data Fig. 11b, owing to an error during figure formatting, the genes listed in the first column shifted down three rows below the first gene on the list, causing a mismatch between the gene names and their characteristics. These errors have been corrected online, and the original Extended Data Fig. 11b is provided as Supplementary Information to this Amendment.

Supplementary Information is available for this Amendment at https://doi. org/10.1038/s41586-018-0232-x. 